\title{
Active Polycondensation of Aromatic Hydroxyl Diesters with Diamines
}

\author{
Naoya Ogata, Kohei Sanui, Kyoichi Kanasugi, \\ and Nobumichi OHIRA \\ Department of Chemistry, Sophia University, \\ 7, Kioi-cho, Chiyoda-ku, Tokyo, Japan.
}

(Received April 23, 1975)

\begin{abstract}
Polycondensation reactions of diesters having phenolic hydroxyl groups with diamines were carried out in order to investigate the enhancement effect of the phenolic hydroxyl group on the polycondensation. Aromatic hydroxyl diesters derived from salicylic acid or hydroquinone had a higher reactivity toward diamines than the reactivity of ordinary diesters. However, their reactivity was inferior to that of aliphatic hydroxyl diesters such as tartarate. The enhancement effect of the phenolic hydroxyl group was presumed to be due to an interaction of the hydroxyl group with the approaching amine.
\end{abstract}

\section{KEY WORDS Polycondensation / Diester / Diamine / Salicylate / Hydroxyl Group /}

Polycondensation reactions of diesters with diamines are usually carried out at elevated temperatures above $200^{\circ} \mathrm{C}$ because of the chemical equilibrium of the polycondensation, while active diesters such as phenyl or thiophenyl esters $^{1,2}$ can be subjected to a polycondensation with diamines under such mild conditions that the polycondensation occurs in solutions even at room temperature. It was found in previous papers that diesters ${ }^{3,4}$ having hetero atoms at positions $\alpha$ or $\beta$ to the carbonyl group or heterocyclic diesters ${ }^{5}$ containing pyridine, furan, or thiophene nuclei could easily react with diamine to form polyamide in solutions under mild conditions.

It was also reported ${ }^{6}$ that aliphatic diesters having hydroxyl groups such as tartarate could react with diamines to form polyamide having pendant hydroxyl groups under mild conditions. The enhancement effect of these hetero atoms or hydroxyl groups is presumed to be ascribed to hydrogen bonding with the approaching amine, so that the local concentration of monomers around the polymer chains increases.

This paper aims at the elucidation of the enhancement effect of hydroxyl groups on the polycondensation of diesters with diamines.
Several aromatic diesters having phenolic hydroxyl groups were synthesized and the polycondensation of these diesters with diamines was carried out.

\section{EXPERIMENTAL}

\section{Syntheses of Aromatic Hydroxyl Diesters}

5,5'-Methylene Disalicylate (MDS). Commercially available 5,5'-methylene disalicylic acid was esterified under reflux in methanol under a stream of hydrogen chloride gas; after evaporating the methanol, the residual product was purified by repeated reprecipitation from benzene$n$-hexane. mp $105^{\circ} \mathrm{C}$. Anal. Calcd: C, 64.56; $\mathrm{H}, 5.06 \%$. Found: C, 65.78; H, 5.10\%.

Diethyl ( $\alpha, \alpha^{\prime}$-Dicyano-2,5-Dihydroxyl)-p-Benzene Diacetate $^{7}(D C D)$. Equimolar amounts of $p$ benzoquinone and $\alpha$-cyano acetate were reacted in aqueous ammonia; the red precipitate formed was purified by recrystallization from a mixture of tetrahydrofuran and ethanol. Yield 47\%, $\mathrm{mp} 288^{\circ} \mathrm{C}$. Anal. Calcd: C, 57.83; H. 4.82; $\mathrm{N}, 8.43 \%$. Found: C, 59.22; H, 4.88; N, $8.87 \%$. 
<smiles></smiles><smiles>CCOC(=O)C(C#N)c1cc(O)c(C(C#N)C(C#N)C(=O)OCC)cc1O</smiles><smiles></smiles>

Dimethyl 2,5-Dihydroxyl-p-Benzene Diacetate ${ }^{7}$ $(D H D)$. DCD was hydrolyzed in aqueous hydrochloric acid under reflux for $8 \mathrm{hr}$; the gray crystal which was obtained after cooling the solution was purified by recrystallization from water. Yield $72 \%, \mathrm{mp} 238^{\circ} \mathrm{C}$.

The product was esterified under reflux in methanol under a stream of hydrogen chloride gas; the white crystal which was obtained from the solution after cooling was recrystallized from methanol. Yield 54\%, mp $221^{\circ} \mathrm{C}$. Anal. Calcd: C, 56.69; H, 5.51, N, 0\%. Found: C, $54.53 ; \mathrm{H}, 5.83, \mathrm{~N}, 0 \%$.<smiles>CCOC(=O)C(C(=O)OCC)c1cc(O)c(C(C#N)C(C#N)C(=O)OCC)cc1O</smiles><smiles>O=C(O)Cc1cc(O)c(CC(=O)O)cc1O</smiles><smiles>COC(=O)Cc1cc(O)c(CC(=O)OCC(C)Cl)cc1O</smiles>

\section{Polycondensation}

Equimolar amounts of aromatic dihydroxyl diesters and diamines were dissolved in various solvents which were previously purified; the solutions were kept with stirring under given conditions. The polycondensation rates were determined by titrating residual amino groups in the solutions with $0.1-N \mathrm{HCl}$ using a pH meter.

\section{Polymer Characterization}

The solution viscosities of the resulting polymers were measured in $m$-cresol or dimethylsulfoxide at $30^{\circ} \mathrm{C}$. The thermal properties of the polymers were determined using the Rigakudenki Differential Thermal and Thermogravimetric Instrument.

\section{RESULTS AND DISCUSSION}

\section{Model Reaction}

Equimolar amounts of methyl salicylate and $n$-butylamine were dissolved in methanol in the concentration of $1 \mathrm{~mol} / l$ and the solution was kept at 30 or $60^{\circ} \mathrm{C}$. Rates of the condensation reaction were measured by titrating residual $n$ butylamine with $0.1-N \mathrm{HCl}$. The results are shown in Figure 1, where the reaction rate of methyl benzoate with $n$-butylamine is compared. It is seen in Figure 1 that methyl salicylate reacted much faster than methyl benzoate; the enhancement effect of phenolic hydroxyl group on the condensation reaction was also recognized. After the condensation reaction was completed, $N$ - $n$-butyl salicylamide (bp $108-110^{\circ} \mathrm{C} / 1 \mathrm{mmHg}$ ) was obtained in an almost quantitative yield.

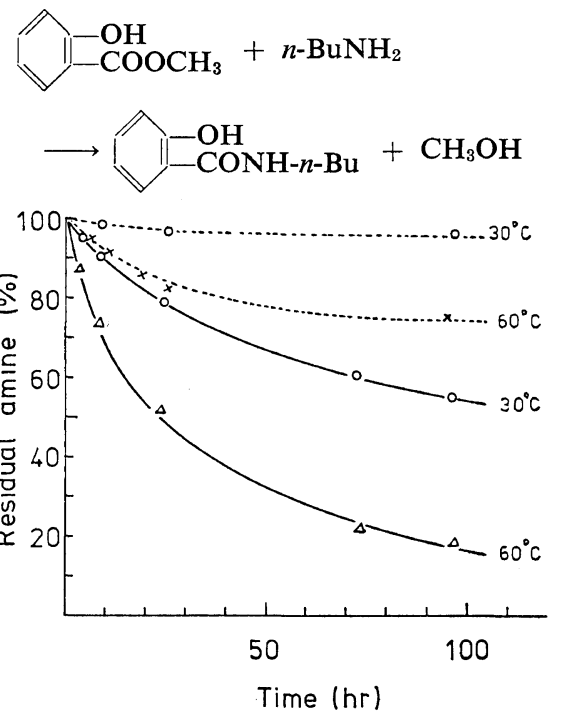

Figure 1. Condensation reaction of methyl salicylate with $n$-butylamine in methanol in the concentration of $1 \mathrm{~mol} / \mathrm{l}$ : _-, methyl salicylate; ----, methyl benzoate. 


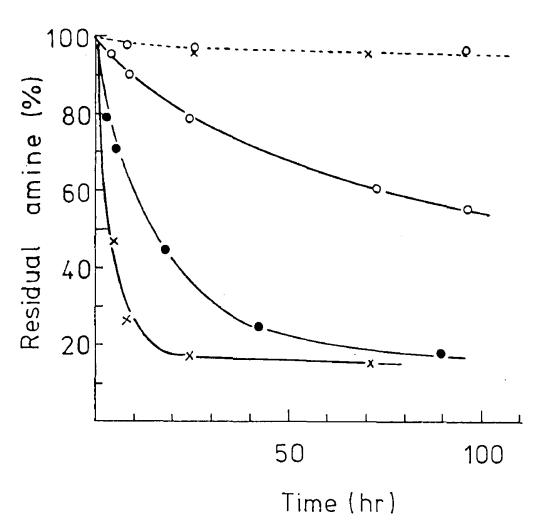

Figure 2. Condensation reaction of methyl salicylate with $n$-butylamine in various solvents at $30^{\circ} \mathrm{C}$ in the concentration of $1 \mathrm{~mol} / l$ : $\times$, HMPA; DMF; $\bigcirc$, methanol; ----, methyl benzoate.

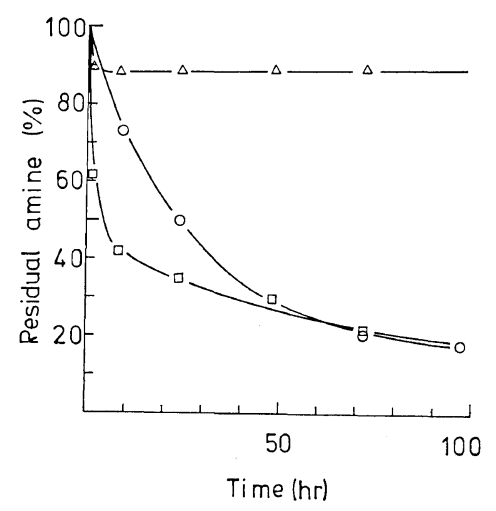

Figure 3. Condensation reaction of methyl $o-, m-$, or $p$-hydroxyl benzoate with $n$-butylamine at $60^{\circ} \mathrm{C}$ in methanol in the concentration of $1 \mathrm{~mol} / \mathrm{l}: \triangle$, $m$-hydroxyl benzoate; $\bigcirc$, o-hydroxyl benzoate; $\square$, p-hydroxyl benzoate.

Rates of the condensation reaction of methyl salicylate with $n$-butylamine, which were determined in various solvents at $30^{\circ} \mathrm{C}$, are shown in Figure 2. A remarkable solvent effect on the condensation reaction was observed. Aprotic polar solvents such as dimethylformamide (DMF) or hexamethylphosphortriamide (HMPA) accelerated the condensation reaction, while no such solvent effect was observed for the condensation reaction of methyl benzoate.

Condensation reactions of $m$ - or $p$-hydroxyl benzoate with $n$-butylamine were carried out in methanol at $60^{\circ} \mathrm{C}$ in the concentration of $1 \mathrm{~mol} / l$. Results are indicated in Figure 3.
It is seen in Figure 3 that the condensation reaction of $p$-hydroxyl benzoate occurred with the fastest rate among three hydroxyl benzoates and $m$-hydroxyl benzoate reacted very slowly with $n$-butylamine. An inductive effect due to the hydroxyl group might have an influence on the condensation reaction of ester with amine.

\section{Polycondensation Reactions}

5,5'-Methylene Disalicylate (MDS). Polycondensation reaction of MDS with hexamethylenediamine (HMD) was carried out in various solvents at $60^{\circ} \mathrm{C}$ in the concentration of 0.1 $\mathrm{mol} / \mathrm{l}$. The results are shown in Figure 4 which indicates that the polycondensation reaction proceeded faster in aprotic polar solvents such as $N$-methyl- $\alpha$-pyrrolidone (NMP), HMPA, DMF, or dimethylsulfoxide (DMSO) than in dioxane or tetrahydrofuran (THF). Figure 4 also indicates that MDS reacted with a much faster rate than dimethyl isophthalate, which is an ordinary aromatic diester. Therefore, it was confirmed that the hydroxyl group attached on an aromatic nucleus had an enhancement effect on the polycondensation reaction of diester with diamine, as is expected from the model reaction. However, the enhancement effect of the phenolic hydroxyl group on the polycondensation reaction was greatly inferior to that of the aliphatic hydroxyl group, since the polycondensation reaction of dimethyl tartarate was com-

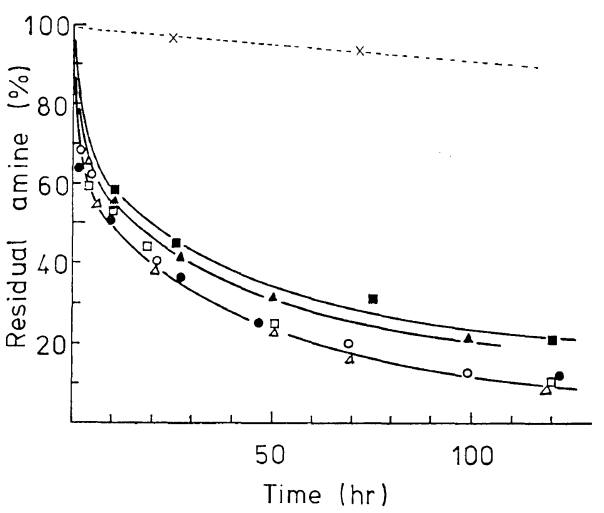

Figure 4. Polycondensation of MDS with HMD in various solvents at $60^{\circ} \mathrm{C}$ in the concentration of $0.1 \mathrm{~mol} / l$ : $\square$, dioxane; $\Delta$, THF; $\bigcirc$, NMP; $\square$, HMPA; $\triangle$, DMSO; ๑, DMF; $\longrightarrow$, MDS; ---, methyl salicylate. 
pleted within several hours, as has been reported in a previous paper. ${ }^{6}$

Figure 5 represents results of the polycondensation reaction of MDS in NMP at various temperatures. Higher temperatures caused faster polycondensation rates and the polycondensation at $100^{\circ} \mathrm{C}$ was completed within one day. Figure 6 shows the polycondensation reaction of MDS with various diamines. HMD and ethylenediamine, which are both aliphatic primary amides, had almost the same reactivity, while piperazine reacted very slowly and aromatic diamine did not react at all under the same

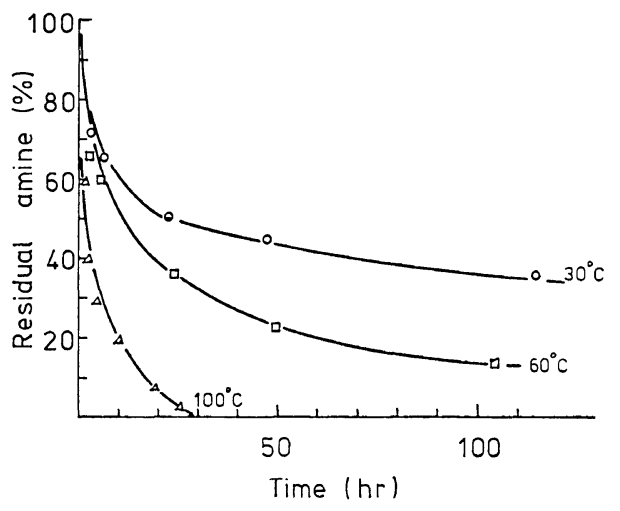

Figure 5. Polycondensation of MDS with HMD in NMP in the concentration of $0.25 \mathrm{~mol} / l$. conditions. The polycondensation of DMS with $m$-xylylenediamine occurred with an intermediate rate between the polycondensation rate with aliphatic diamines and that with piperazine. These results suggest that a steric interaction might occur between MDS and the approaching amine, although the basicity of the amino group is one of the factors for the reactivity order.

Polymers were recovered by pouring the solutions into excess acetone. Results are sum-

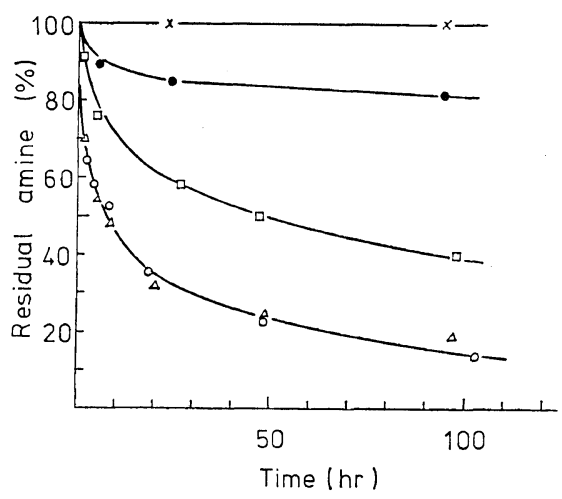

Figure 6. Polycondensation of MDS with various diamines in NMP at $60^{\circ} \mathrm{C}$ in the concentration of $0.25 \mathrm{~mol} / l: \quad \times, \quad p$-phenylenediamine; $\square, \quad m$ xylylenediamine; $\bullet$, piperazine; $\triangle$, ethylenediamine; $\bigcirc$, HMD.

Table I. Polycondensation of MDS with diamines in NMPa

\begin{tabular}{|c|c|c|c|c|c|c|}
\hline \multirow{2}{*}{ Diamine } & \multirow{2}{*}{ Additive $^{b}$} & \multirow{2}{*}{$\underset{{ }^{\circ} \mathrm{C}}{\text { Temp }}$} & \multirow{2}{*}{$\begin{array}{c}\text { Time, } \\
\text { Day }\end{array}$} & \multicolumn{3}{|c|}{ Polymer } \\
\hline & & & & Yield, \% & $\eta_{\mathrm{inh}^{d}}$ & $\underset{\substack{\text { Dec temp, } \\
{ }^{\circ}}}{ }$ \\
\hline HMD & None & 30 & 56 & $96^{c}$ & 0.10 & 220 \\
\hline HMD & None & 60 & 21 & 97 & 0.09 & 220 \\
\hline HMD & None & 100 & 2 & 93 & 0.08 & 220 \\
\hline HMD & $\mathrm{ZnCl}_{2}$ & 60 & 21 & 54 & 0.09 & 220 \\
\hline HMD & $\mathrm{CaCl}_{2}$ & 60 & 21 & 54 & 0.08 & 220 \\
\hline HMD & $\mathrm{FeCl}_{3}$ & 60 & 21 & 36 & 0.05 & 222 \\
\hline HMD & $\mathrm{LiCl}$ & 60 & 21 & 36 & 0.06 & 220 \\
\hline HMD & $\mathrm{H}_{3} \mathrm{PO}_{4}$ & 60 & 21 & 36 & 0.05 & 220 \\
\hline Ethylenediamine & None & 60 & 40 & 75 & 0.07 & 200 \\
\hline$m$-Xylylenediamine & None & 60 & 40 & 69 & 0.05 & 230 \\
\hline Piperazine & None & 60 & 50 & 28 & 0.07 & 200 \\
\hline
\end{tabular}

a Monomer concn, $0.25 \mathrm{~mol} / \mathrm{l}$.

b $5 \mathrm{~mol} \%$.

c Anal. Calcd: C, 68.45; H, 6.58; N, 7.60\%. Found: C, 67.70; H, 6.52, N, 6.97\%.

d Measured in $m$-cresol at $30^{\circ} \mathrm{C}$. 
marized in Table I. All polymers were identified as the corresponding polyamides from elemental and infrared analyses. Polyamide was obtained from MDS and HMD in a quantitative yield, but the solution viscosities were not satisfactory, as indicated in Table I. The polyamide from MDS and HMD had no distinct melting point and it decomposed on heating at around $220^{\circ} \mathrm{C}$. Inorganic salts such as zinc chloride or calcium chloride, which had a great accelerating effect on the polycondensation of aliphatic hydroxyl diesters such as dimethyl tartarate, ${ }^{6}$ rather inhibited the polycondensation reaction. The reason for this might be ascribed to the chelation of these inorganic salts with the phenolic hydroxyl group, which might result in the masking of the hydroxyl group. The reason of low solution viscosities of resulting polyamides is not clear yet; it may be related to the conformation of the resulting polyamides in solutions, which may disturb the amine approaching near to the polymer chain ends. More detailed studies on the reaction mechanism are still necessary to prove the enhancement effect of the hydroxyl group on the polycondensation reaction. However, a suggested explanation for the enhancement effect is based on an interaction of the hydroxyl group with the approaching amine by forming strong hydrogen bonds.

2,5-Dihydroxyl-p-Benzene Diacetate (DHD). The polycondensation reaction of DHD with HMD was carried out in DMSO or HMPA at
30 or $60^{\circ} \mathrm{C}$ in the concentration of $0.1 \mathrm{~mol} / l$. Results are indicated in Figure 7. When the polycondensation of DHD was compared with that of MDS, both DHD and MDS reacted with HMD with almost the same rate in DMSO, as can be seen in Figure 7. Therefore, the enhancement effect of the phenolic hydroxyl group was still strongly observed even when the ester group was separated from the hydroxyl group by an intermediate unit of one methylene group from the aromatic nucleus.

Neither acceleration nor any inhibition effect on the polycondensation of DHD were observed

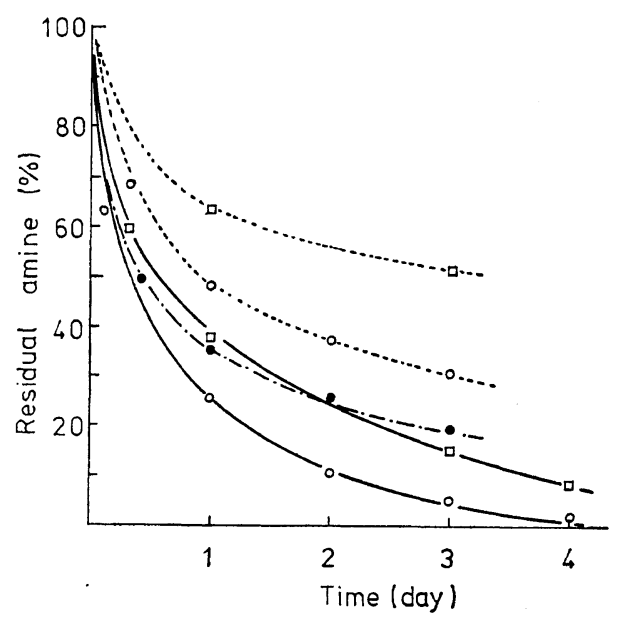

Figure 7. Polycondensation of dimethyl DHD with HMD in the concentration of $0.1 \mathrm{~mol} / \mathrm{l}: \square$, HMPA; $\bigcirc$, DMSO; ----, $30^{\circ} \mathrm{C}$; - $60^{\circ} \mathrm{C}$; -.MDS polycondensation in NMP at $60^{\circ} \mathrm{C}$.

Table II. Polycondensation of DHD with HMD

\begin{tabular}{|c|c|c|c|c|c|c|}
\hline \multirow{2}{*}{ Solvent } & \multirow{2}{*}{ Additive $^{b}$} & \multirow{2}{*}{$\begin{array}{c}\text { Concn, } \\
\mathrm{mol} / \mathrm{l}\end{array}$} & \multirow{2}{*}{$\underset{{ }^{\circ} \mathrm{C}}{\text { Temp }}$} & \multirow{2}{*}{$\begin{array}{c}\text { Time, } \\
\text { Day }\end{array}$} & \multicolumn{2}{|c|}{ Polymer } \\
\hline & & & & & Yield, \% & $\eta_{\text {inh }}{ }^{c}$ \\
\hline DMSO & None & 0.1 & 30 & 30 & 76 & 0.13 \\
\hline DMSO & None & 0.1 & 60 & 30 & 59 & 0.23 \\
\hline DMSO & $\mathrm{FeCl}_{3}$ & 0.1 & 60 & 30 & 77 & 0.12 \\
\hline DMSO & $\mathrm{CH}_{3} \mathrm{OLi}$ & 0.1 & 60 & 30 & 25 & - \\
\hline DMSO & $\mathrm{H}_{3} \mathrm{PO}_{4}$ & 0.1 & 60 & 30 & 61 & 0.11 \\
\hline DMSO & None & 0.25 & 60 & 30 & 70 & 0.14 \\
\hline HMPA & None & 0.1 & 30 & 30 & 55 & 0.08 \\
\hline HMPA & None & 0.1 & 60 & 30 & 62 & 0.11 \\
\hline
\end{tabular}

a Dimethyl 2,5-dihydroxyl-p-benzene diacetate.

b $5 \mathrm{~mol} \%$.

c Measured in DMSO at $30^{\circ} \mathrm{C}$. 


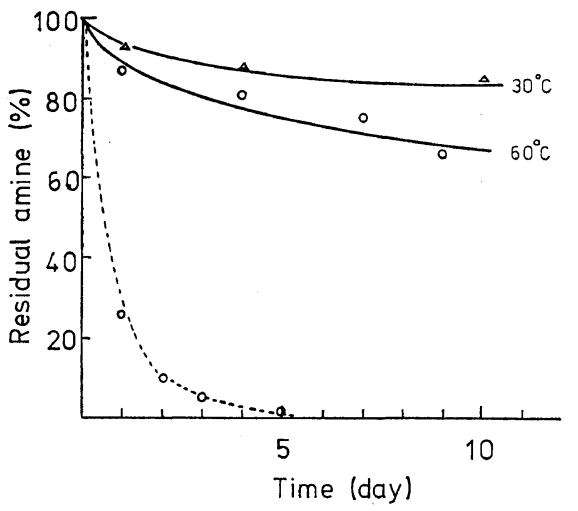

Figure 8. Polycondensation of DCD with HMD in DMSO in the concentration of $0.1 \mathrm{~mol} / l$ at 30 or $60^{\circ} \mathrm{C}$ : ---, MDS polycondensation at $60^{\circ} \mathrm{C}$ in DMSO.

for ferric chloride or phosphoric acid when they were added to the solutions. Polymers were collected by pouring the solutions into excess acetone, followed by filtering and drying. Polymers were identified as the corresponding polyamides from DHD and HMD by elemental and infrared analyses. Results are summarized in Table II, where it is seen that solution viscosities of resulting polyamide from DHD are slightly superior to those of polyamide from MDS. The polyamide from DHD and HMD had no distinct melting point and it decomposed on heating at around $180^{\circ} \mathrm{C}$.

The polycondensation reaction of diethyl $\left(\alpha, \alpha^{\prime}\right.$ dicyano-2,5-dihydroxyl)-p-benzene diacetate (DCD) with HMD was carried out in DMSO at 30 or $60^{\circ} \mathrm{C}$ in the concentration of $0.1 \mathrm{moll}$. Results are indicated in Figure 8. It was found that the polycondensation of DCD occurred with a much slower rate than that of MDS and that DCD had almost the same reactivity as that of ordinary diesters such as dimethyl isophthalate. The retardation in the reactivity of DCD might be related with the electron-accepting nitrile groups attached on the $\alpha$-methylene groups of the diester. The absorption due to the nitrile group of DCD appeared at $2200 \mathrm{~cm}^{-1}$ with a very weak intensity in its infrared spectrum and the absorption owing to ester carbonyl group appeared at $1640 \mathrm{~cm}^{-1}$, which indicates that the carbonyl group of DCD is strongly polarized. Therefore, it is assumed that the nitrile group may have an intramolecular interaction with the adjacent phenolic hydroxyl group in such a way that the hydroxyl group may be masked by the nitrile group, as shown below<smiles>CCOC(=O)C(/C=N\N)c1ccccc1O</smiles>

This presumption of the masking effect due to the adjacent nitrile group of DCD also supports the explanation of the enhancement effect of the hydroxyl group on the polycondensation reaction, which is based on the formation of hydrogen bonding of the hydroxyl group with the approaching amine so as to increase the local concentration of monomers around the polymer chains.

\section{REFERENCES}

1. N. Ogata, K. Sanui, and K. Iijima, J. Polym. Sci., Polymer Chem. Ed., 11, 1095 (1973).

2. N. Ogata and S. Okamoto, ibid., 11, 2537 (1973).

3. N. Ogata, K. Sanui, and K. Okouchi, Polymer J., 5, 186 (1973).

4. N. Ogata, Y. Hosoda, and G. Suzuki, ibid., 6, 412 (1974).

5. N. Ogata and K. Shimamura, ibid., 7, 72 (1975).

6. N. Ogata and Y. Hosoda, J. Polym. Sci., Polym. Letters Ed., 12, 355 (1974).

7. E. C. Horning, Ed., "Organic Syntheses," Coll. Vol. 3, John Wiley \& Sons, Inc., New York, N.Y., 1955, p 286. 